\title{
The optical head of the EnVisS camera for the Comet Interceptor ESA mission: phase 0 study
}

Da Deppo, Vania, Pernechele, Claudio, Jones, Geraint, Brydon, George, Zuppella, Paola, et al.

Vania Da Deppo, Claudio Pernechele, Geraint H. Jones, George Brydon, Paola Zuppella, Paolo Chioetto, Simone Nordera, Alessandra Slemer, Giuseppe Crescenzio, Emanuele Piersanti, Paolo Spanò, Gino Bucciol, Luca Consolaro, Luisa Lara, Andris Slavinskis, "The optical head of the EnVisS camera for the Comet Interceptor ESA mission: phase 0 study," Proc. SPIE 11443, Space Telescopes and Instrumentation 2020: Optical, Infrared, and Millimeter Wave, 1144379 (13 December 2020); doi: 10.1117/12.2562907 


\title{
The optical head of the EnVisS camera for the Comet Interceptor ESA mission: Phase 0 study
}

\author{
Vania Da Deppo*a,b, Claudio Pernechele ${ }^{\mathrm{b}}$, Geraint H. Jones ${ }^{\mathrm{c}, \mathrm{d}}$, George Brydon ${ }^{\mathrm{c}, \mathrm{d}}$, Paola Zuppella, ${ }^{\mathrm{a}, \mathrm{b}}$, \\ Paolo Chioetto ${ }^{\mathrm{a}, \mathrm{b}, \mathrm{e}}$, Simone Nordera ${ }^{\mathrm{a}, \mathrm{b}}$, Alessandra Slemer ${ }^{\mathrm{a}, \mathrm{b}}$, Giuseppe Crescenzio ${ }^{\mathrm{f}}$, Emanuele \\ Piersanti $^{\mathrm{f}}$, Paolo Spanò ${ }^{\mathrm{f}}$, Gino Bucciol ${ }^{\mathrm{f}}$, Luca Consolaro ${ }^{\mathrm{g}}$, Luisa Lara ${ }^{\mathrm{h}}$, Andris Slavinskis ${ }^{\mathrm{i}}$ \\ ${ }^{a}$ CNR-Institute for Photonics and Nanotechnologies Padova, Via Trasea 7, 35131 Padova, Italy \\ ' INAF-Osservatorio Astronomico di Padova, Vicolo dell'Osservatorio 5, 35122 Padova, Italy \\ ${ }^{\mathrm{C}} \mathrm{UCL}$, Mullard Space Science Laboratory, Holmbury St. Mary, Dorking, Surrey RH5 6NT, UK \\ ${ }^{\mathrm{d}}$ The Centre for Planetary Sciences UCL/Birkbeck, Gower Street, London, WC1E 1BT, UK \\ ${ }^{\mathrm{e} C I S A S}$ G. Colombo, University of Padova, Via Venezia 15, 35131 Padova, Italy

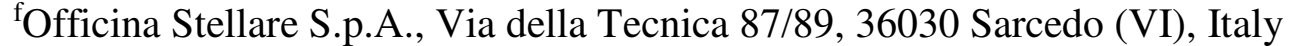 \\ 'Lobre S.r.1, Via Antonio Meucci 6, 25013 Carpenedolo (BS), Italy \\ ${ }^{\mathrm{h}}$ CSIC-Instituto de Astrofisica de Andalusia, c/ Glorieta de la Astronomia s/n, 18008 Granada, Spain \\ ${ }^{\mathrm{i}}$ Aalto University, School of Electrical Engineering, 02150 Espoo, Finland
}

\begin{abstract}
EnVisS (Entire Visible Sky) is an all-sky camera specifically designed to fly on the space mission Comet Interceptor. This mission has been selected in June 2019 as the first European Space Agency (ESA) Fast mission, a modest size mission with fast implementation.
\end{abstract}

Comet Interceptor aims to study a dynamically new comet, or interstellar object, and its launch is scheduled in 2029 as a companion to the ARIEL mission.

The mission study phase, called Phase 0, has been completed in December 2019, and then the Phase A study had started. Phase A will last for about two years until mission adoption expected in June 2022.

The Comet Interceptor mission is conceived to be composed of three spacecraft: spacecraft A devoted to remote sensing science, and the other two, spacecraft B1 and B2, dedicated to a fly-by with the comet.

EnVisS will be mounted on spacecraft B2, which is foreseen to be spin-stabilized. The camera is developed with the scientific task to image, in push-frame mode, the full comet coma in different colors. A set of ad-hoc selected broadband filters and polarizers in the visible range will be used to study the full scale distribution of the coma gas and dust species.

The camera configuration is a fish-eye lens system with a FoV of about $180^{\circ} \times 45^{\circ}$.

This paper will describe the preliminary EnVisS optical head design and analysis carried out during the Phase 0 study of the mission.

Keywords: space camera, optical head design, all-sky imager, Comet Interceptor

\section{INTRODUCTION}

The Comet Interceptor mission aims to encounter and explore a truly pristine comet, i.e. an object very likely entering the inner Solar System for the first time, or, possibly, an interstellar object originating at another star [1][2]. Due to the extremely high orbital eccentricities of either type of target, Comet Interceptor will perform a flyby like Giotto rather than a rendezvous like Rosetta. Nevertheless, the mission is scientifically compelling and combines the first exploration of a new type of target, as was the case for Giotto, with unique measurements that go beyond what Rosetta achieved, in some areas, within the constraints of the F class missions.

*vania.dadeppo@pd.ifn.cnr.it; phone +39-049 9815639

Space Telescopes and Instrumentation 2020: Optical, Infrared, and Millimeter Wave, edited by Makenzie Lystrup, Marshall D. Perrin, Proc. of SPIE Vol. 11443, 1144379 - @ 2020 SPIE

CCC code: $0277-786 \mathrm{X} / 20 / \$ 21 \cdot$ doi: $10.1117 / 12.2562907$ 
The Comet Interceptor mission will involve separate spacecraft elements working together to ensure a low-risk, bountiful, interdisciplinary scientific return through unprecedented multi-point measurements. Multiple viewing positions will greatly increase the 3D information provided on the target and its jets/coma. Similarly, in situ observations of the cometary environment also benefit from multiple sampling paths. The multiple elements can sample gas composition and density, dust flux, and plasma and solar wind interactions, to build up a 3D 'snapshot' of the region around the target.

One spacecraft, named spacecraft A, will make remote and upstream in situ observations of the target from afar, to protect it from the dust environment of an active comet, and act as the primary communications hub with Earth for all other mission elements. Two other spacecraft, B1 and B2, will be deployed to venture closer to the target, carrying complementary instrument payloads, to build up a 3D picture of the comet. This approach will also enable a combination of a low risk and guaranteed baseline science return from the more distant spacecraft with higher-risk but high-gain sampling of the inner coma by the releasable probes, which do not necessarily need to survive the full encounter for mission success.

A and B2 will be provided by the European Space Agency, while B1 by the Japan Aerospace Exploration Agency (JAXA).

\section{THE ENVISS CAMERA}

On the B2 spacecraft, one of the two probes foreseen to venture near the comet to perform a fly-by, an all sky-imager is foreseen to be mounted. The entire visible sky (EnVisS) camera is designed to study the coma of the comet. Both dust and gas emissions are expected to be imaged by the instrument. Being mounted on a spinning spacecraft, the camera will acquire at a high rate slices of the sky that will be later stitched together to obtain a full sky coverage. See Figure 1(a) for the image acquisition scheme and (b) for a simulated full-sky reconstructed image. In Figure 1(b), the image's $\mathrm{x}$ and $\mathrm{y}$ axes cover $360^{\circ}$ and $180^{\circ}$ respectively, and an example of the saturation of detector columns due to the presence of Sun in the FoV is also simulated.

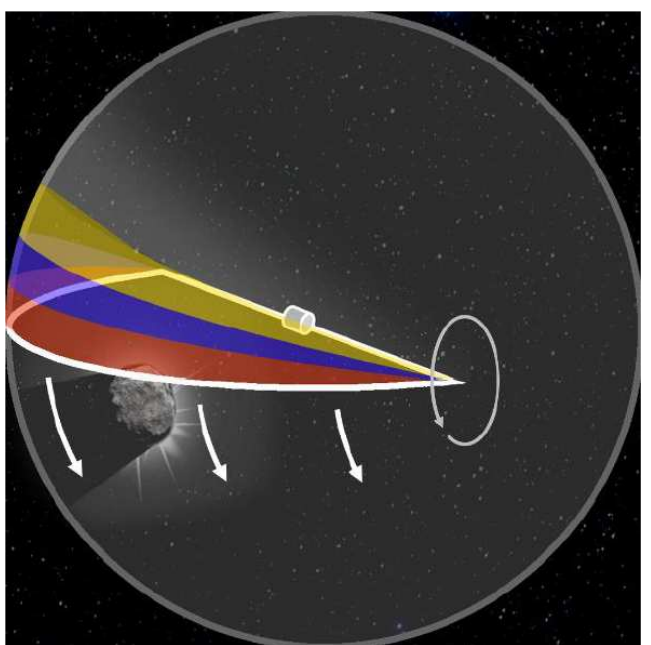

(a)

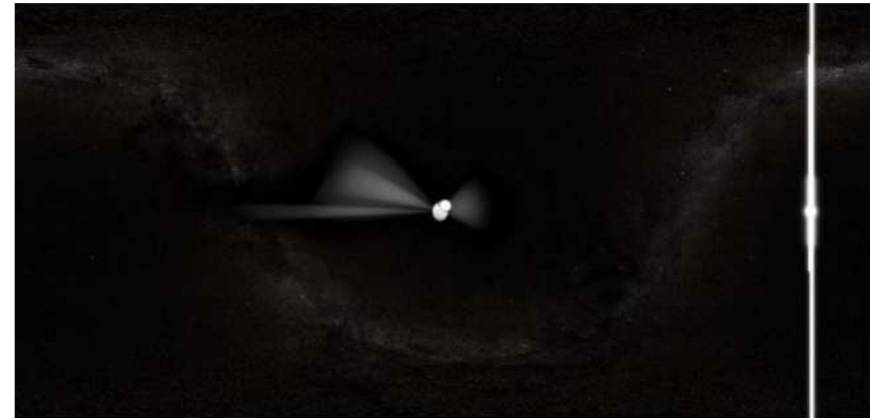

(b)

Figure 1. In (a) illustration of EnVisS' full-sky spin scanning. Coloured strips represent the instrument's multiple scan lines which build up full sky images as the spacecraft rotates. In (b) Example radiometrically simulated EnVisS image of comet nucleus and dust jets during flyby [3].

\subsection{Background}

To acquire images in several spectral ranges simultaneously while the spacecraft is orbiting around the target, mainly the Earth, or a planetary surface, the push-broom scan imaging scheme is adopted by many space instruments [4] [5]. The push-frame imaging technique can also be considered in some cases for multispectral imaging with filter strips directly bonded to the detector or mounted very near to it [6] [7]. This latter choice allows for a compact, low mass and low complexity camera to be used.

For cameras mounted on spinning spacecraft, a rotational push-broom, or push-frame, scheme imaging can be adopted to scan and image the whole scene around the spacecraft. The across track FoV should be in this case as high as possible, ideally $180^{\circ}$, to cover in one rotation the full sky. 
An example of an instrument that sweeps nearly the entire sky as the spacecraft moves through its orbit was the Solar Mass Ejection Imager (SMEI) aboard the Coriolis spacecraft designed to detect and forecast the arrival of coronal mass ejections [8]. This type of instruments feature high wide angle fish eye optics.

\subsection{Optical design requirements}

EnVisS should provide a quasi-total coverage of the sky as seen from the B2 spacecraft. This feature will provide information on the comet's entire environment, whilst the photogrammetry permitted by the spacecraft's changing perspective will allow the study of the neutral gas, dust and plasma components of the coma's three-dimensional structure.

The camera has the opportunity to view a diverse range of scenes as the spacecraft approaches, enters, traverses and departs the comet's environment. Images captured throughout the flyby will give views from a wide range of phase angles, and record the evolution of the dust environment.

To measure and analyze the coma constituents in multiple spectral ranges and different polarisation orientations, spectral and polarimetric filters have to be considered. The use of scan rather than frame imaging allows data to be collected through multiple different optical filters simultaneously, without the need for a filter wheel. Different adjacent filter strips can be inserted in the focal plane assembly, just in front of the detector. This concept simplifies the instrument's operation, avoids the challenges involved with filter changes during a rapid flyby, and allows to design a compact, low mass instrument.

A summary of scientific requirements and optical characteristics expected for the camera are reported respectively in Table 1(a) and (b). Eight filters are currently planned and their selection has been guided by Rosetta OSIRIS findings. The overall required global wavelength coverage of the camera is between $600 \mathrm{~nm}$ and $800 \mathrm{~nm}$ expected to be reached with diffraction limited performance.

The spatial resolution is about $0.1^{\circ}$ per pixel sufficient for highly valuable observations of the sometimes-subtle structuring in a coma's multiple constituents. The FoV of the instrument has to be of $180^{\circ}$ across track and $45^{\circ}$ along track to allow for 7-8 filters strips to be accommodated; each filter strip should cover a $5^{\circ} \mathrm{FoV}$ and some gaps should be taken into account to prevent cross-talk and straylight issues that are well known to happen in such kind of filter arrangements [9].

Table 1 (a) EnVisS scientific requirements; (b) EnVisS optical characteristics.

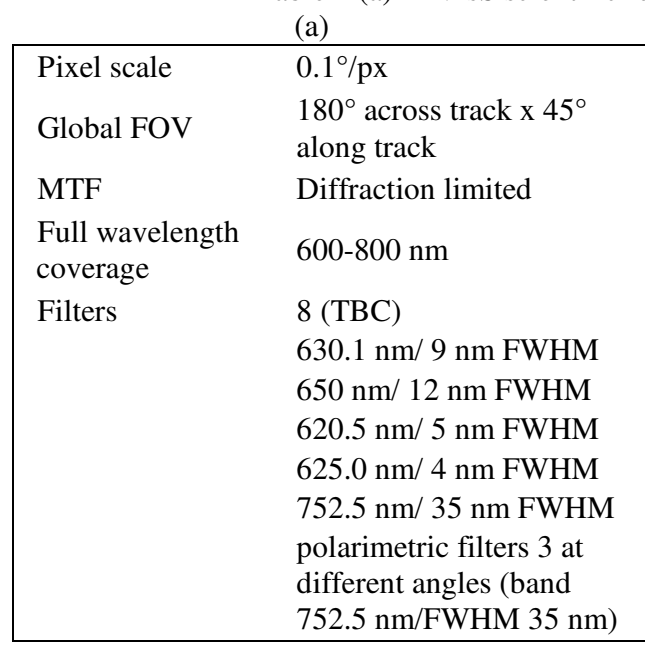

(b)

\begin{tabular}{|c|c|}
\hline Optical concept & Fish eye \\
\hline $\begin{array}{l}\text { Paraxial focal } \\
\text { length }\end{array}$ & $3.4 \mathrm{~mm}$ \\
\hline $\begin{array}{l}\text { Pupil size } \\
\text { (diameter) }\end{array}$ & $1.2 \mathrm{~mm}$ \\
\hline Focal ratio & $F / 2.8$ \\
\hline Mean image scale & $0.12 \%$ px \\
\hline $\begin{array}{l}\text { Geometrical } \\
\text { distortion }\end{array}$ & $<4 \%$ (F-Theta distortion law) \\
\hline Detector & $\begin{array}{l}\text { APS with rolling shutter (format: } \\
1504 \times 2000 ; 7.0 \mu \mathrm{m} \text { squared pixel) }\end{array}$ \\
\hline
\end{tabular}

An equidistant F-Theta fisheye optical system using space qualified rad-hard glass has been considered for the EnVisS optical head design [10]. The fisheye lens has its heritage in a hyper hemispheric lens already designed for space [11][12] and optical parameters of such an extremely wide angle lens, anamorphism and distortion, have been already studied in detail [13][14].

Since B2 is expected to rotate at 15 r.p.m. a full revolution will happen in 4 seconds, thus expected integration times for each filter strip are of the order of $1 \mathrm{~ms}$, with a repetition time of about 50-60 ms.

The position of the camera on the spacecraft B2 will be such that during the flyby the camera and its baffle are shielded from dust impacts thanks to the dust shield. This means that a small region centered on the forward direction of $\mathrm{B} 2$ and 
around the spin axis will be hidden by the shield, this part will be imaged by the other camera present on B2: the OPIC camera [15], thus guaranteeing the full-sky coverage.

\subsection{Focal plane assembly}

As stated in the previous paragraph, the EnVisS design includes a set of 7 to 8 filter strips, 4 filters have been selected with bands appropriate for observations of specific gas and ion emission lines and for detecting the continuum diffused by the comet dust particles, some 3 to 4 strips will have polarimetric capabilities to study the comet light polarization. The detector considered for the Phase 0 Comet Interceptor study was the one used for JUICE JANUS imager, i.e. the E2V CIS115. This sensor is a back-side illuminated CMOS with 1504 × 2000 pixels and $7 \mu \mathrm{m}$ pitch, a peak quantum efficiency $>90 \%$, a fullwell of $27000 \mathrm{e}$ - and is featuring a rolling shutter reading mode. Full resolution push-frame images will measure $4000 \mathrm{x}$ 2000 pixels; one such frame is created for each waveband.

The expected schematics of the filter strips images on the detector is shown in Figure 2.

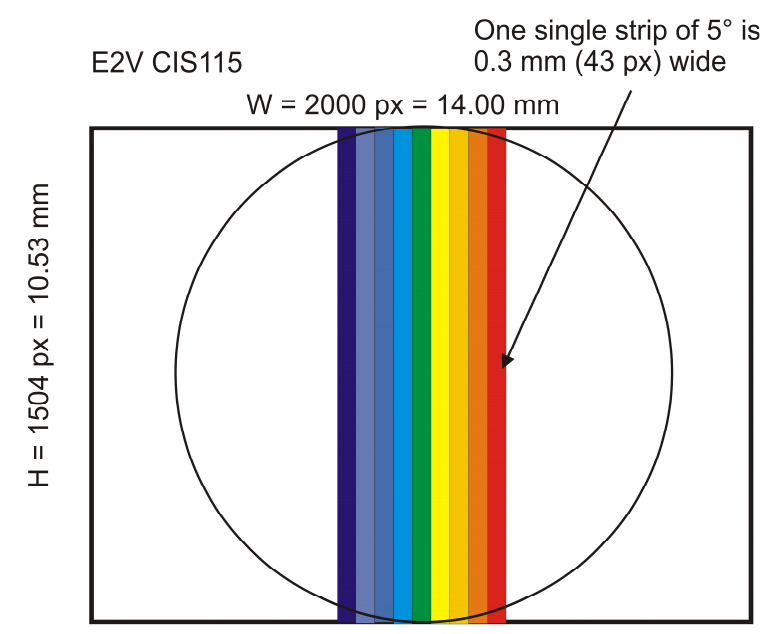

Figure 2. Schematics of the filter strips on the selected detector.

Due to some availability issues with the E2V detector, at present a 3D-plus camera equipped with a CMV4000 detector has been chosen as the baseline detector package for EnVisS.

\section{ENVISS OPTICAL HEAD PERFORMANCE}

\subsection{Preliminary mechanical design}

Different solutions have been taken into account for the mechanical design of the EnVisS optical head. Eventually, a solution with a barrel in Titanium alloy realized by several independent cells with a front stage including the two external lenses, the biggest ones, has been proposed as the baseline. Titanium has been judged an optimal choice because of the thermal matching with the glass considered for the lenses [16]. In Figure 3 (a) and (b), the optical head mechanical concept is depicted, in the first image the section view allows to clearly see the barrel with the cells and the outer piece holding the external lenses, in the second one a global view of the whole design is presented including a sketch of the proposed baffle elements. The external baffle is the main element to stray light suppression. The baffle has been tentatively designed starting from literature examples [6].

\subsection{Focus stability}

The MTF drops of about a factor of $50 \%$ in a focus range of about $\pm 25 \mu \mathrm{m}$. However, the size of the instrument and the materials used for its design (Titanium and glass) guarantee the system is very stable with respect to thermal variations.

In a range of -40 to $+60{ }^{\circ} \mathrm{C}$ the optical system has an elongation from $101.96 \mathrm{~mm}$ to $102.59 \mathrm{~mm}$. A more accurate thermal analysis, including glass refractive index variation with temperature, must be carried out to understand the actual focus stability.

Air to vacuum image plane shift is $-17 \mu \mathrm{m}$ : the focal plane moves toward the last lens. This must be considered if the system is going to be aligned and/or tested in air. 


\subsection{Preliminary tolerance analysis}

A first quick run for sensitivity shows that the system is feasible. Tolerances have been studied taking into account four compensation interfaces: the lenses are divided into groups to be integrated into cells as per the mechanical design. The cells are then meant to be assembled together to form the full instrument. The four interfaces will be joined by shims, the appropriate size of the shims to be used during alignment will be defined later when the project will be more advanced. Typical tolerances at a first run are about $50 \mu \mathrm{m}$ for centration and thickness of air and glass; 1.5 arcmin for lenses tilts; $0.5 \%$ on radii of curvature, surface quality is assumed to be $\lambda / 10$ and glasses are considered to be standard graded.

A first Montecarlo, on a dozen samples to size the tolerance study, shows no sudden drop in MTF so no major issues are identified, however a detailed study is necessary to better understand the optical performance of the "as-built" system.

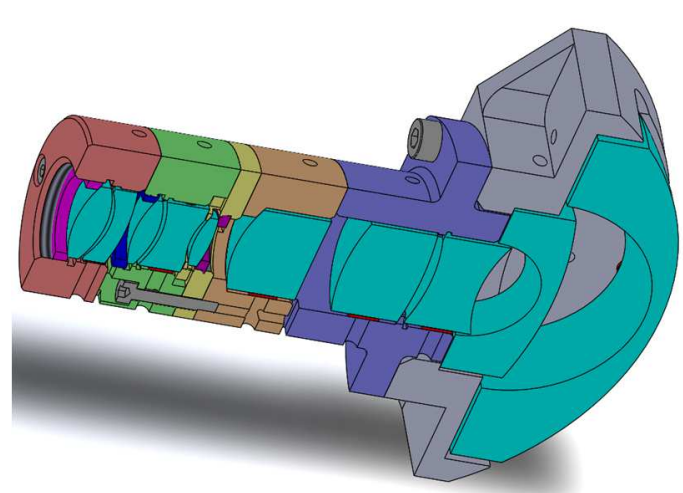

(a)

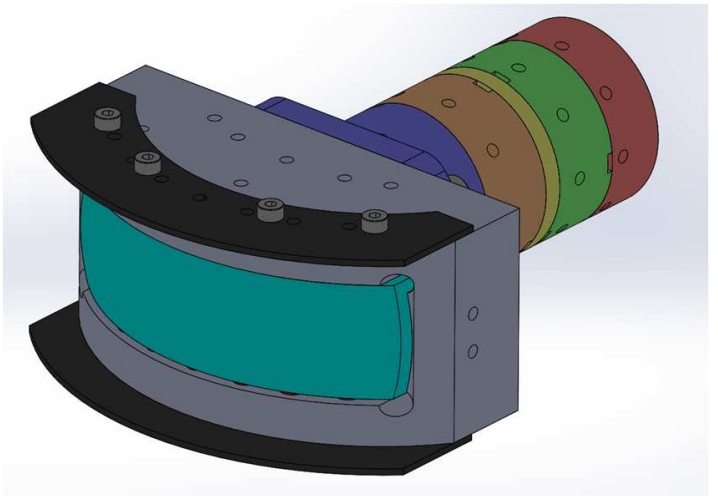

(b)

Figure 3 Preliminary EnVisS optical head mechanical design. (a) section; (b) full view, including the proposed baffle design.

\section{CONCLUSIONS}

In this paper the preliminary design and performance of the optical head of the EnVisS camera for the Comet Interceptor ESA mission have been presented. The work described has been carried out during the Phase 0 study of the mission.

EnVisS is an all-sky camera specifically designed to be mounted on the B2 spacecraft which is a spin stabilized probe that will performance a fly-by with the mission target, i.e. a pristine comet or interstellar object. The scientific requirements and the solutions adopted for the design of the camera optical head have been highlighted.

Thanks to the push-frame acquisition technique and to the focal plane concept, featuring filter strips mounted right in front of the detector, the system mass and envelope can be maintained as low as possible compatible with the resources available for an F-class mission on a probe spacecraft like B2.

Some preliminary analysis on focus stability and tolerance has also been considered to assess the feasibility of the design and its expected performance in-flight.

The design will be further studied and adapted in the following phase of the mission study.

\section{ACKNOWLEDGMENTS}

This activity has been conducted and funded through an Agenzia Spaziale Italiana (ASI) contract to the Istituto Nazionale di Astrofisica (INAF) n. 2020-4-HH.0.

\section{REFERENCES}

[1] Snodgrass, C. and Jones, G. H., "The European Space Agency's Comet Interceptor lies in wait", Nat. Commun. 10, 5418 (2019). 
[2] Jones, G. H., Snodgrass, C. and the Comet Interceptor Consortium, "Comet Interceptor: A proposed ESA Mission to a Dynamically New Comet", EPSC Abstracts Vol. 13, EPSC-DPS2019-1679-1, (2019).

[3] Brydon, G. and Jones, G. H., "Comet Interceptor's EnVisS Camera: Multispectral and Polarimetric Full-sky Imager for a Comet Flyby", EPSC Abstracts Vol. 13, EPSC-DPS2019-1691-1, (2019).

[4] Jaumann, R. et al., "The high-resolution stereo camera (HRSC) experiment on Mars Express: Instrument aspects and experiment conduct from interplanetary cruise through the nominal mission", Planet. Space Sci. 55(7-8), 928-952, (2007).

[5] Robinson, M. S., et al., "Lunar Reconnaissance Orbiter Camera (LROC) Instrument Overview", Space Sci. Rev. $150,81-124,(2010)$.

[6] Bell, J. F., III, et al., "Mars Reconnaissance Orbiter Mars Color Imager (MARCI): Instrument description, calibration and performance", J. Geophys. Res. 114, E08S92, (2009).

[7] Da Deppo, V., Naletto, G., Cremonese, G. and Calamai, L., "Optical design of the single-detector planetary stereo camera for the BepiColombo European Space Agency mission to Mercury", App. Opt. 49(15), 2910-9, (2010).

[8] Eyles, C. J., et al., "The Solar Mass Ejection Imager (SMEI)", Solar Physics 217, 319-347 (2003).

[9] Da Deppo, V., Cremonese, G. and Naletto, G., "Ghost images determination for the stereoscopic imaging channel of SIMBIOSYS for the BepiColombo ESA mission", Proc. SPIE 8167, Optical Design and Engineering IV, $81671 \mathrm{U}(2011)$.

[10] Pernechele, C., Da Deppo, V., Brydon, G., Jones, G. H., Lara, L. and Michaelis, H., "Comet interceptor's EnVisS camera sky mapping function", Proc. SPIE 11203, Advances in Optical Astronomical Instrumentation 2019, $112031 \mathrm{M}(2020)$.

[11]Pernechele, C., et al., "Hyper hemispheric lens applications in small and micro satellites", Adv. Space Res. 62, 3449-3461 (2018).

[12] Opromolla, R., et al., "A new star tracker concept for satellite attitude determination based on a multi-purpose panoramic camera", Acta Astron. 140, 166-175 (2017).

[13] Pernechele, C. and Villa, F., "Hyper-hemispheric lens distortion model for 3D-imaging SPAD-array-based applications". Proc. SPIE, 9626 (2015).

[14] Pernechele, C., "Hyper hemispheric lens", Optics Express 24, 5014-5019 (2016).

[15] Pajusalu, M., et al., "Developing autonomous image capturing systems for maximum science yield for high flyby velocity small solar system body exploration", 71st International Astronautical Congress, IAC-20-A3.4B.4 (2020).

[16] Pernechele, C., et al., "Telecentric F-Theta Fisheye Lens for Space Application", submitted to OSA continuum (2020). 